\title{
Coordination of Power and Natural Gas Systems: Convexification Approaches for Linepack Modeling
}

Schwele, Anna; Ordoudis, Christos; Kazempour, Jalal; Pinson, Pierre

Published in:

Proceedings of IEEE PES PowerTech 2019

Link to article, DOI:

10.1109/PTC.2019.8810632

Publication date:

2020

Document Version

Peer reviewed version

Link back to DTU Orbit

Citation $(A P A)$ :

Schwele, A., Ordoudis, C., Kazempour, J., \& Pinson, P. (2020). Coordination of Power and Natural Gas Systems: Convexification Approaches for Linepack Modeling. In Proceedings of IEEE PES PowerTech 2019 IEEE. https://doi.org/10.1109/PTC.2019.8810632

\section{General rights}

Copyright and moral rights for the publications made accessible in the public portal are retained by the authors and/or other copyright owners and it is a condition of accessing publications that users recognise and abide by the legal requirements associated with these rights.

- Users may download and print one copy of any publication from the public portal for the purpose of private study or research.

- You may not further distribute the material or use it for any profit-making activity or commercial gain

- You may freely distribute the URL identifying the publication in the public portal 


\title{
Coordination of Power and Natural Gas Systems: Convexification Approaches for Linepack Modeling
}

\author{
Anna Schwele, Christos Ordoudis, Jalal Kazempour, Pierre Pinson \\ Center for Electric Power and Energy, Technical University of Denmark, Kgs. Lyngby, Denmark \\ $\{$ schwele, chror, seykaz, ppin\}@elektro.dtu.dk
}

\begin{abstract}
Utilizing operational flexibility from natural gas networks can foster the integration of uncertain and variable renewable power production. We model a combined power and natural gas dispatch to reveal the maximum potential of linepack, i.e., energy storage in the pipelines, as a source of flexibility for the power system. The natural gas flow dynamics are approximated by a combination of steady-state equations and varying incoming and outgoing flows in the pipelines to account for both natural gas transport and linepack. This steadystate natural gas flow results in a nonlinear and nonconvex formulation. To cope with the computational challenges, we explore convex quadratic relaxations and linear approximations. We propose a novel mixed-integer second-order cone formulation including McCormick relaxations to model the bidirectional natural gas flow accounting for linepack. Flexibility is quantified in terms of system cost compared to a dispatch model that either neglects linepack or assumes infinite storage capability.

Index Terms-Combined power and natural gas dispatch, convexification, McCormick relaxation, second-order cone program, steady-state gas flow.
\end{abstract}

\section{NOMENCLATURE}

Sets

$\mathcal{I} \quad$ Set of dispatchable power plants $i$.

$\mathcal{C} \quad$ Subset of dispatchable power plants excluding natural gas-fired ones $(\mathcal{C} \subset \mathcal{I})$.

$\mathcal{G} \quad$ Subset of natural gas-fired power plants $(\mathcal{G} \subset \mathcal{I})$.

$\mathcal{J} \quad$ Set of wind power units $j$.

$\mathcal{T} \quad$ Set of time periods $t$.

$\mathcal{L} \quad$ Set of electricity transmission lines $(n, r)$.

$\mathcal{N} \quad$ Set of electricity network nodes $n$.

$\mathcal{K} \quad$ Set of natural gas supply units $k$.

$\mathcal{Z} \quad$ Set of natural gas pipelines $(m, u)$.

$\mathcal{M} \quad$ Set of natural gas network nodes $m$.

$\mathcal{V} \quad$ Set of fixed pressure points $v$.

$\mathcal{A}_{n}^{(.)} \quad$ Set of assets located at electricity network node $n$.

$\mathcal{A}_{m}^{(.)} \quad$ Set of assets located at natural gas network node $m$.

$\Theta \quad$ Set of optimization variables.

\section{Variables}

$p_{i, t}, w_{j, t}$ Dispatch of units $i$ and $j$ in period $t$, respectively [MW].

$g_{k, t} \quad$ Dispatch of unit $k$ in period $t[\mathrm{kcf} / \mathrm{h}]$.

$\theta_{n, t} \quad$ Voltage angle at node $n$ in period $t$ [rad].

$f_{n, r, t} \quad$ Power flow in line $(n, r)$ in period $t$ [MW].

$\mathrm{pr}_{m, t} \quad$ Pressure at node $m$ in period $t$ [psig].

$h_{m, u, t} \quad$ Average mass of natural gas (linepack) in pipeline $(m, u)$ in period $t[\mathrm{kcf}]$.

$q_{m, u, t}^{\text {in/out }} \quad$ Inflow/outflow natural gas rate of pipeline $(m, u)$ in period $t[\mathrm{kcf} / \mathrm{h}]$.

$q_{m, u, t} \quad$ Natural gas flow in pipeline $(m, u)$ in period $t[\mathrm{kcf} / \mathrm{h}]$.

$q_{m, u, t}^{+/-} \quad$ Natural gas flow in pipeline $(m, u)$ from node $m$ to $u /$ from node $u$ to $m$ in period $t[\mathrm{kcf} / \mathrm{h}]$.

$y_{m, u, t} \quad$ Binary variable defining the direction of the natural gas flow in pipeline $(m, u)$ in period $t\{0,1\}$.

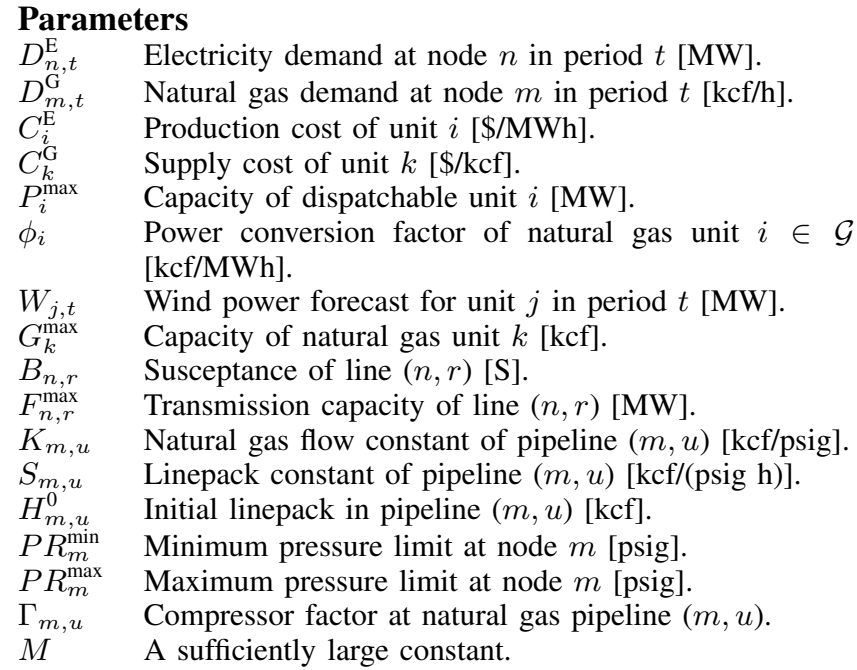

\section{INTRODUCTION}

The growing share of stochastic renewable energy sources that introduce uncertainty and variability into the operation of power system increases the need for flexible resources. Natural gas-fired power plants (NGFPP) typically provide operational flexibility in systems with high share of renewable energy penetration [1], thus linking the power and natural gas systems [2]. In power system operations, security and network constraints related to the natural gas system are becoming important for generation scheduling [3], unit commitment [4], and reserve scheduling decisions [5] to ensure fuel availability for NGFPPs. Apart from ensuring technical feasibility of shortterm operations, accounting for the natural gas network unlocks its inherent flexibility in order to facilitate the integration of renewables and deal with uncertainties and variability from large-scale wind power penetration [6], [7]. This necessitates a proper coordination between power and natural gas systems.

Several works in the literature have investigated different coordination levels of power and natural gas systems in terms of short-term operational integration, proving the advantages of such coupling for the whole system, see [6]-[9]. A full coupling (co-optimization) of the two energy systems is not compatible with the current market regulations in most countries which usually require decoupled operations on different temporal scales [10]. The full coupling requires a single entity to operate the whole integrated system. This is the case in Denmark, where Energinet.dk is operating both systems, but separately [11]. The co-optimization model yields an ideal 
benchmark showing the maximum potential flexibility that can be unlocked, obtaining the minimum overall cost for the two systems [12]. This benchmark, though not directly implementable in practice, is highly useful, since it provides a lower bound for system cost, and can be used to assess the performance of any other coordination scheme that aligns with current market regulations.

The ability to store natural gas in the pipelines is referred to as linepack, and can provide short-term flexibility to the power system. Unlocking linepack flexibility requires accurate modeling of natural gas flow dynamics along pipelines as well as efficient solution methods. Different integrated natural gas and power flow models are presented in [8] using dynamic natural gas flow models, while [13] models steady-state gas flow constraints. It is worth mentioning that modeling bidirectional gas flow is crucial, since the direction of natural gas flow in the pipeline may change due to sudden off-take from NGFPPs to compensate for variability from renewable generation.

Several works have aimed at convexifying the steady-state natural gas flow via linear approximation or convex relaxation techniques. Reference [14] introduces a semidefinite programming (SDP) relaxation for the natural gas flow, assuming that the flow direction is known. Similarly, [15] proposes a secondorder cone (SOC) relaxation for modeling optimal power and natural gas flows, assuming fixed flow directions and ignoring compressors, linepack and storage. A mixed-integer secondorder cone programming (MISOCP) relaxation is proposed in [16] to represent natural gas flows for expansion planning of natural gas and power systems with unidirectional gas flow, while the expansion planning model in [17] allows for varying flow directions along a pipeline. Reference [18] proposes a MISOCP relaxation model treating gas flow direction in each pipeline as a variable, proving the uniqueness and exactness of the solution, but ignoring linepack. The piecewise linear approximation model in [19] also considers bidirectional gas flows but neglects linepack. The mixed-integer linear programming (MILP) models proposed in [6] and [7] yield improved flexibility and reliability when accounting for the bidirectional gas flows and linepack. More specifically, the models in [6] and [7] are able to incorporate linepack dynamics while allowing for bidirectional natural gas flow using an outer linear approximation and a piecewise linear approximation, respectively. Table I summarizes the convexification approaches for steady-state natural gas flow models in the literature, and compares them with the proposed model in this paper.

To the best of our knowledge, the existing quadratic and semidefinite relaxation models either assume known flow directions or neglect the ability of pipelines to store natural gas, see [14]-[18]. Thus, we propose a novel MISOCP model including McCormick relaxations of the steady-state natural gas flow to incorporate both linepack and bidirectional flow. Similar to the approach in [20], which investigates the linepack flexibility provided by district heating networks for the power system, our work evaluates the social value of short-term operational flexibility that the natural gas network can provide to the electricity system. The objective of this paper is to present
TABLE I

COMPARISON OF NATURAL GAS FLOW CONVEXIFICATION APPROACHES

\begin{tabular}{clll}
\hline Reference & Natural gas flow model & Bidirectional flow & Linepack \\
\hline$[14]$ & Relaxation (SDP) & No & No \\
{$[15]$} & Relaxation (SOCP) & No & No \\
{$[16]$} & Relaxation (MISOCP) & No & No \\
{$[17],[18]$} & Relaxation (MISOCP) & Yes & No \\
{$[19]$} & Approximation (MILP) & Yes & No \\
{$[6],[7]$} & Approximation (MILP) & Yes & Yes \\
This paper & Relaxation (MISOCP) & Yes & Yes \\
\hline
\end{tabular}

a methodology for a combined electricity and natural gas dispatch as an ideal benchmark that appropriately models the dynamics of gas flow. We use this model to assess the ability of the natural gas network to react to changes in the power system due to intermittent renewables. In order to evaluate the modeling of the linepack properties to account for inherent flexibility of the natural gas system, we compare the proposed MISOCP relaxation of the bidirectional nonconvex steadystate natural gas flow under different levels of wind penetration to the MILP approximation proposed in [6]. Finally, the value of linepack flexibility provided by the gas network is quantified in terms of system cost compared to the coupled dispatch of the electricity and natural gas systems either without linepack modeling or with infinite storage capability.

The paper is organized as follows. Section II introduces the formulation of the combined power and natural gas dispatch model. In Section III, we explore two convexification approaches for efficiently solving the natural gas flow model, which are compared in Section IV for a case study quantifying the value of natural gas network flexibility for the power system. Section V draws the conclusion.

\section{COMBINED POWER AND NATURAL GAS DISPATCH}

\section{A. Model framework and assumptions}

We propose a fully coupled electricity and natural gas dispatch that co-optimizes the operations in both systems. Both wind power production and wind spillage costs are assumed to be zero. Since the focus of this paper is modeling the flexibility provided by the natural gas network, we aim at accurately modeling the gas flow problem, while we use a lossless DC model for the power flows on the electricity side. Under the assumption of isothermal natural gas flow in horizontal pipelines, the natural gas flow is represented by its steady-state gas flow equation. The dynamics in pipelines are approximated by accounting for the energy storage capacity of gas networks in the form of linepack through varying inand outflows. Compressor stations are modeled with a constant compressor factor neglecting fuel and power consumption of compressors [3].

\section{B. Co-optimization Model}

The original combined dispatch problem, which is mixedinteger and nonconvex, is formulated by the following optimization problem (1):

$$
\min _{\Theta} \sum_{t \in \mathcal{T}}\left(\sum_{i \in \mathcal{C}} C_{i}^{\mathrm{E}} p_{i, t}+\sum_{k \in \mathcal{K}} C_{k}^{\mathrm{G}} g_{k, t}\right)
$$


subject to

$$
\begin{aligned}
& 0 \leq p_{i, t} \leq P_{i}^{\max }, \forall i, t, \\
& 0 \leq w_{j, t} \leq W_{j, t}, \forall j, t \text {, } \\
& f_{n, r, t}=B_{n, r}\left(\theta_{n, t}-\theta_{r, t}\right), \forall(n, r) \in \mathcal{L}, t \\
& -F_{n, r}^{\max } \leq f_{n, r, t} \leq F_{n, r}^{\max }, \forall(n, r) \in \mathcal{L}, t \\
& -\pi \leq \theta_{n, t} \leq \pi, \forall n, t, \theta_{n, t}=0, \forall n: \text { ref }, t \\
& \sum_{i \in \mathcal{A}_{n}^{I}} p_{i, t}+\sum_{j \in \mathcal{A}_{n}^{J}} w_{j, t}-\sum_{(n, r) \in \mathcal{L}} f_{n, r, t}=D_{n, t}^{\mathrm{E}}, \forall n, t, \\
& 0 \leq g_{k, t} \leq G_{k}^{\max }, \forall k, t \text {, } \\
& P R_{m}^{\min } \leq p r_{m, t} \leq P R_{m}^{\max }, \forall m, t \text {, } \\
& p r_{u, t} \leq \Gamma_{m, u} p r_{m, t}, \forall(m, u) \in \mathcal{Z}, t, \\
& q_{m, u, t}\left|q_{m, u, t}\right|=K_{m, u}^{2}\left(p r_{m, t}^{2}-p r_{u, t}^{2}\right), \forall(m, u) \in \mathcal{Z}, t \text {, } \\
& q_{m, u, t}=q_{m, u, t}^{+}-q_{m, u, t}^{-}, \forall(m, u) \in \mathcal{Z}, t, \\
& 0 \leq q_{m, u, t}^{+} \leq M y_{m, u, t}, \forall(m, u) \in \mathcal{Z}, t, \\
& 0 \leq q_{m, u, t}^{-} \leq M\left(1-y_{m, u, t}\right), \forall(m, u) \in \mathcal{Z}, t \text {, } \\
& q_{m, u, t}^{+}=\frac{q_{m, u, t}^{\text {in }}+q_{m, u, t}^{\text {out }}}{2}, \forall(m, u) \in \mathcal{Z}, t, \\
& q_{m, u, t}^{-}=\frac{q_{u, m, t}^{\text {in }}+q_{u, m, t}^{\text {out }}}{2}, \forall(m, u) \in \mathcal{Z}, t, \\
& h_{m, u, t}=S_{m, u} \frac{p r_{m, t}+p r_{u, t}}{2}, \forall(m, u) \in \mathcal{Z}, t, \\
& h_{m, u, t}=h_{m, u,(t-1)}+q_{m, u, t}^{\text {in }}-q_{m, u, t}^{\text {out }}+q_{u, m, t}^{\text {in }}-q_{u, m, t}^{\text {out }} \text {, } \\
& \forall(m, u) \in \mathcal{Z}, t>1 \text {, } \\
& h_{m, u, t}=H_{m, u}^{0}+q_{m, u, t}^{\text {in }}-q_{m, u, t}^{\text {out }}+q_{u, m, t}^{\text {in }}-q_{u, m, t}^{\text {out }}, \\
& \forall(m, u) \in \mathcal{Z}, t=1 \text {, } \\
& H_{m, u}^{0} \leq h_{m, u, t}, \forall(m, u) \in \mathcal{Z}, t=|\mathcal{T}| \\
& \sum_{k \in \mathcal{A}_{m}^{K}} g_{k, t}-\sum_{i \in \mathcal{A}_{m}^{G}} \phi_{i} p_{i, t} \\
& -\sum_{u:(m, u) \in \mathcal{Z}}\left(q_{m, u, t}^{\text {in }}-q_{u, m, t}^{\text {out }}\right)=D_{m, t}^{\mathrm{G}}, \forall m, t,
\end{aligned}
$$

where the set of optimization variables is $\Theta=\left\{q_{m, u, t}, y_{m, u, t}\right.$, $q_{m, u, t}^{+}, q_{m, u, t}^{-}, q_{m, u, t}^{\text {in }}, q_{m, u, t}^{\text {out }}, h_{m, u, t}, p r_{m, t}, g_{k, t}, p_{i, t}, w_{j, t}, \theta_{n, t}$, $\left.f_{n, r, t}\right\}$. Objective function (1a) minimizes the total cost of operating the power and natural gas systems, i.e., the production cost of non-NGFPPs and natural gas supply cost. The model contains two sets of constraints: (1b)-(1g) correspond to the power system while $(1 \mathrm{~h})-(1 \mathrm{u})$ pertain to the natural gas system. The two systems are coupled through (1u) translating the dispatch of NGFPPs into a time-varying nodal demand for natural gas by $\sum_{i \in \mathcal{A}_{m}^{G}} \phi_{i} p_{i, t}, \forall m, t$. Constraints (1b) and (1c) impose capacity limits of the power plants and wind farms, respectively. Constraints (1d) and (1e) define power flows along each transmission line and bound them to transmission capacity. In (1f) the voltage angles are restricted and that of the reference node is set to zero. Constraint (1g) enforces the power balance at each node. Constraints (1h) and (1i) impose limits on capacity of natural gas supply and nodal pressure, respectively. Pipelines with compressors relate the inlet and outlet pressures between two adjacent nodes via

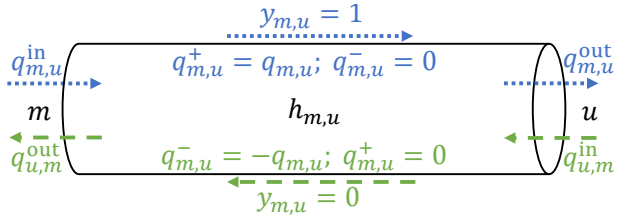

Fig. 1. Bidirectional flow along a pipeline (time index $t$ is dropped for notational clarity)

compressor factor $\Gamma_{m, u}$ in $(1 \mathrm{j})$. The piecewise quadratic and nonconvex constraint $(1 \mathrm{k})$, known as Weymouth equation, describes the steady-state gas flow between nodes $m$ and $u$, which depends on the pressure at the adjacent nodes and the physical properties of the pipeline reflected in parameter $K_{m, u}$. The natural gas flow in each pipeline is decomposed in (11) to two non-negative variables $q_{m, u, t}^{+}$and $q_{m, u, t}^{-}$, which are required to determine the direction of flow using binary variable $y_{m, u, t}$ in $(1 \mathrm{~m})$ and $(1 \mathrm{n})$. Note that the big constant $M$ is set to the maximum gas flow along a pipeline to ensure that either $(1 \mathrm{~m})$ or $(1 \mathrm{n})$ is active. The flow in each pipeline is defined as the average of inflow and outflow according to (1o) and (1p), see Fig. 1. The average mass in each pipeline is given by (1q) which determines the linepack on pipelines based on pressures at adjacent nodes and parameter $S_{m, u}$ reflecting the pipeline properties. The mass conservation at each pipeline is enforced by (1r). Constraint (1s) sets the initial amount of linepack, while (1t) imposes a minimum linepack level at the end of time period to ensure that the natural gas in the network is not depleted. Finally, constraint (1u) imposes the nodal natural gas balance, and couples the power and natural gas systems through fuel consumption of NGFPPs.

Note that the resulting mixed-integer and nonlinear programming (MINLP) model (1) is computationally challenging, since there is currently no off-the-shelf solver to efficiently solve such a problem. In the next section, we investigate the two options to convexify the nonlinearities of this model.

\section{LINEPACK CONVEXIFICATION APPROACHES}

We first propose a novel MISOCP relaxation that allows for modeling both bidirectional natural gas flow and convexified dynamics of linepack in pipelines. Then, for comparison purposes, another convexification alternative based on a linear approximation method proposed in [6] is briefly explained.

\section{A. Proposed MISOCP Relaxation}

Weymouth equation $(1 \mathrm{k})$ can be equivalently written as:

$$
\begin{aligned}
& q_{m, u, t}\left|q_{m, u, t}\right| \leq K_{m, u}^{2}\left(p r_{m, t}^{2}-p r_{u, t}^{2}\right), \forall(m, u) \in \mathcal{Z}, t, \\
& q_{m, u, t}\left|q_{m, u, t}\right| \geq K_{m, u}^{2}\left(p r_{m, t}^{2}-p r_{u, t}^{2}\right), \forall(m, u) \in \mathcal{Z}, t .
\end{aligned}
$$

We drop (2b) and then replace (2a) by the following set of constraints in (3). Compared to (2a), note that the absolute function is omitted in (3) but the bidirectional flows along pipelines are still modeled by additional terms related to binary variable $y_{m, u, t}[18]$.

$$
\left\{-M\left(1-y_{m, u, t}\right) \leq q_{m, u, t} \leq M y_{m, u, t},\right.
$$




$$
\begin{aligned}
& q_{m, u, t}^{2} \leq K_{m, u}^{2}\left(p r_{m, t}^{2}-p r_{u, t}^{2}\right)+M^{2}\left(1-y_{m, u, t}\right) \\
& \left.q_{m, u, t}^{2} \leq K_{m, u}^{2}\left(p r_{u, t}^{2}-p r_{m, t}^{2}\right)+M^{2} y_{m, u, t},\right\} \\
& \forall(m, u) \in \mathcal{Z}, t
\end{aligned}
$$

While (3a) is a linear constraint, (3b) and (3c) are still not in the form of SOC constraints. We introduce two auxiliary variables $\varphi_{m, u, t}^{+}=p r_{m, t}+p r_{u, t}$ and $\varphi_{m, u, t}^{-}=p r_{m, t}-p r_{u, t}$, so that $\varphi_{m, u, t}^{+} \varphi_{m, u, t}^{-}=p r_{m, t}^{2}-p r_{u, t}^{2}$. Accordingly, we reformulate $(3 \mathrm{~b})$ and $(3 \mathrm{c})$ as

$$
\begin{aligned}
& \left\{q_{m, u, t}^{2} \leq K_{m, u}^{2}\left(\varphi_{m, u, t}^{+} \varphi_{m, u, t}^{-}\right)+M^{2}\left(1-y_{m, u, t}\right)\right. \\
& \left.q_{m, u, t}^{2} \leq K_{m, u}^{2}\left(-\varphi_{m, u, t}^{+} \varphi_{m, u, t}^{-}\right)+M^{2} y_{m, u, t},\right\} \\
& \forall(m, u) \in \mathcal{Z}, t
\end{aligned}
$$

Similarly, constraints (1j) and (1q) in the original problem are replaced by

$$
\begin{gathered}
\left\{\frac{1}{2}\left(\varphi_{m, u, t}^{+}-\varphi_{m, u, t}^{-}\right) \leq \Gamma_{m, u} \frac{1}{2}\left(\varphi_{m, u, t}^{+}+\varphi_{m, u, t}^{-}\right),\right. \\
\left.h_{m, u, t}=S_{m, u} \frac{\varphi_{m, u, t}^{+}}{2},\right\} \forall(m, u) \in \mathcal{Z}, t .
\end{gathered}
$$

Since $p r_{m, t}$ is substituted by auxiliary variables, we drop the pressure bounds (1i), and then introduce parameters $\underline{\varphi}_{m, u, t}^{+}=$ $P R_{m}^{\min }+P R_{u}^{\min }, \bar{\varphi}_{m, u, t}^{+}=P R_{m}^{\max }+P R_{u}^{\max }, \underline{\varphi}_{m, u, t}^{-}=P R_{m}^{\min }-$ $P R_{u}^{\max }$, and $\bar{\varphi}_{m, u, t}^{-}=P R_{m}^{\max }-P R_{u}^{\min }$ to limit those auxiliary variables as

$$
\begin{aligned}
\left\{\underline{\varphi}_{m, u, t}^{+}\right. & \leq \varphi_{m, u, t}^{+} \leq \bar{\varphi}_{m, u, t}^{+}, \\
\underline{\varphi}_{m, u, t}^{-} & \left.\leq \varphi_{m, u, t}^{-} \leq \bar{\varphi}_{m, u, t}^{-},\right\} \forall(m, u) \in \mathcal{Z}, t .
\end{aligned}
$$

We still need to rephrase (4a) and (4b) due to the bilinear term $\varphi_{m, u, t}^{+} \varphi_{m, u, t}^{-}$. Therefore, we use McCormick relaxation technique by introducing a new auxiliary variable, denoted as $\psi_{m, u, t}$, and defining the McCormick envelopes [21]. For notional clarity, we drop subscripts $m, u$ and $n$ from all variables and parameters. These envelopes are:

$$
\begin{aligned}
& \psi \geq \underline{\varphi}^{+} \varphi^{-}+\varphi^{+} \underline{\varphi}^{-}-\underline{\varphi}^{+} \underline{\varphi}^{-} \\
& \psi \geq \bar{\varphi}^{+} \varphi^{-}+\varphi^{+} \bar{\varphi}^{-}-\bar{\varphi}^{+} \bar{\varphi}^{-} \\
& \psi \leq \bar{\varphi}^{+} \varphi^{-}+\varphi^{+} \underline{\varphi}^{-}-\bar{\varphi}^{+} \underline{\varphi}^{-} \\
& \psi \leq \underline{\varphi}^{+} \varphi^{-}+\varphi^{+} \bar{\varphi}--\underline{\varphi}^{+} \bar{\varphi}^{-} .
\end{aligned}
$$

Now, (4a) and (4b) are expressed by mixed-integer secondorder cone constraints:

$$
\begin{aligned}
& \left\{q_{m, u, t}^{2} \leq K_{m, u}^{2} \psi_{m, u, t}+M^{2}\left(1-y_{m, u, t}\right),\right. \\
& \left.q_{m, u, t}^{2} \leq-K_{m, u}^{2} \psi_{m, u, t}+M^{2} y_{m, u, t},\right\} \\
& \forall(m, u) \in \mathcal{Z}, t .
\end{aligned}
$$

Note that if $y_{m, u, t}=0$, then (8a) is satisfied trivially, while (8b) becomes $q_{m, u, t}^{2} \leq-K_{m, u}^{2} \psi_{m, u, t}$. Similarly, (8b) is nonbinding when $y_{m, u, t}=1$, while $(8 \mathrm{~b})$ reads as $q_{m, u, t}^{2} \leq$ $K_{m, u}^{2} \psi_{m, u, t}$. The resulting MISOCP model includes (1a)-(1h), (11)-(1p), (1r)-(1u), (3a), and (5)-(8).

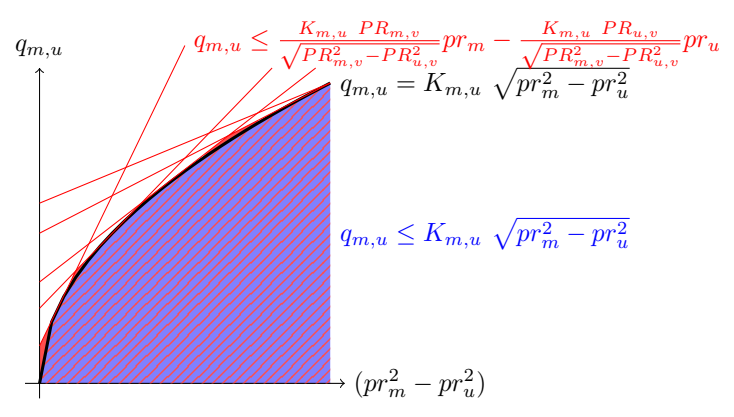

Fig. 2. SOC relaxation and outer linear approximation of (1k)

\section{B. MILP Approximation}

We compare the proposed MISOCP relaxation to the outer approximation approach based on the Taylor series expansion around fixed pressure points from [6]. This tractable MILP formulation of bidirectional gas flow model accounts for linepack flexibility. Instead of relaxing the solution space described by the Weymouth equation into a SOC as in (2a), the approach in [6] defines a number of planes tangent to the cone described by (1k), see Fig. 2. Each equality constraint (1k) is replaced by a set of linear inequalities (9), that linearly approximate the Weymouth equation around fixed pressure points $P R_{m, v}, P R_{u, v}, \forall(m, u) \in \mathcal{Z}, v \in \mathcal{V}$ :

$$
\begin{aligned}
& q_{m, u, t}^{+} \leq \frac{K_{m, u} P R_{m, v}}{\sqrt{P R_{m, v}^{2}-P R_{u, v}^{2}}} p r_{m, t}-\frac{K_{m, u} P R_{u, v}}{\sqrt{P R_{m, v}^{2}-P R_{u, v}^{2}}} p r_{u, t} \\
& +M\left(1-y_{m, u, t}\right), \forall\{(m, u) \in \mathcal{Z} \mid m>u\}, v, t, \\
& q_{m, u, t}^{-} \leq \frac{K_{m, u} P R_{u, v}}{\sqrt{P R_{u, v}^{2}-P R_{m, v}^{2}}} p r_{u, t}-\frac{K_{m, u} P R_{m, v}}{\sqrt{P R_{u, v}^{2}-P R_{m, v}^{2}}} p r_{m, t} \\
& +M y_{m, u, t}, \forall\{(m, u) \in \mathcal{Z} \mid m<u\}, v, t .
\end{aligned}
$$

Table II summarizes the introduced models. Note that the solutions obtained from both MISOCP and MILP approaches may not be necessarily feasible for the original model (1). However, these models are still useful as benchmarks to assess the value of linepack flexibility.

\section{NUMERICAL STUdY}

We compare the convexification approaches presented in Section III using an integrated energy system consisting of a 12-node natural gas system and the IEEE 24-node reliability test system [6]. The forecast profiles of total electricity load and wind power production over the 24-hour scheduling horizon are illustrated in Fig. 3. We solve all models using an Intel CoreTM i7-7820HQ with four processors clocking at 2.70 GHz and $16 \mathrm{~GB}$ of RAM in Python using Gurobi solver

TABLE II

SUMMARY OF COMBINED POWER AND NATURAL GAS DISPATCH MODELS

\begin{tabular}{ll}
\hline Model & Properties \\
\hline MINLP & Original model; no off-the-shelf solver \\
MISOCP & Quadratic relaxation of the original MINLP model \\
MILP & Linear approximation of the original MINLP model \\
\hline
\end{tabular}




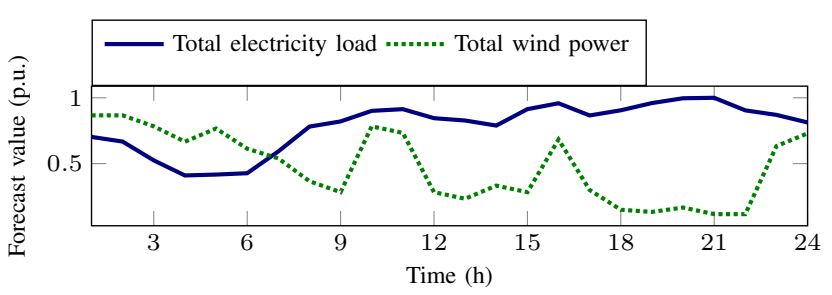

Fig. 3. Demand and wind power forecasts

package 7.5.2. All input data and codes used are available in [22].

We now present our results. Fig. 4 shows the total system cost, which is the optimal value obtained for (1a), under different levels of wind power penetration, i.e., the total installed wind power capacity divided by total demand of power system. We first quantify the value of modeling the linepack by comparing the total system cost obtained in MISOCP and MILP models with that of a case neglecting linepack (green curve in Fig. 4). This comparison indicates that the flexibility revealed from the pipelines decreases the total system cost. This cost reduction in MISOCP and MILP models is $2 \%$ and $1 \%$ on average across different levels of wind penetration, respectively. In order to quantify the amount of flexibility unveiled from linepack, we now compare the results achieved with a case including an ideal electricity storage. This storage has infinite capacity, charging and discharging rates allowing for the most cost-efficient shift of the electricity demand over hours. According to a performance ratio defined

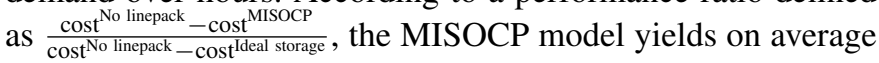
a cost saving equal to $25.5 \%$ of that in the ideal storage case, while this value is $13.1 \%$ for the MILP model.

For the case of 50\% wind penetration, Fig. 5 shows the daily profiles of total charging and discharging of energy in the natural gas pipelines as well as the total amount of natural gas supplied and consumed by NGFPPs. The total linepack increases from the initial 526,600 kcf to maximum $570,193 \mathrm{kcf}$ and 572,578 kcf in the MISOCP and MILP models, respectively. For the two convexification approaches the charging and discharging profiles of the natural gas network are slightly different, see Figs. 5(a) and 5(b). Compared to the case neglecting linepack (Figs. 5(c) and 5(f)), both approaches result in shifting the natural gas demand profile in a more cost-

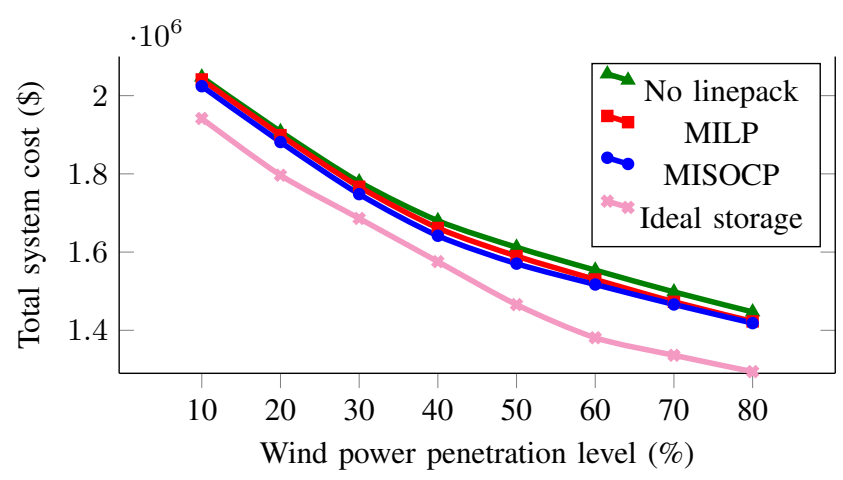

Fig. 4. Total system cost as a function of wind power penetration efficient manner and thus, utilizing less expensive natural gas supply for electricity production (Figs. 5(d) and 5(e)). The amount of energy stored in the pipelines directly impacts the profiles of natural gas supply and consumption by NGFPPs, see Figs. 5(d)-5(f). According to these plots, the gas supply curve in the model neglecting linepack is highly volatile (Fig. 5(f)), while it is entirely flat in the MISOCP model (Fig. $5(d)$ ). This profile is also flat during the most hours of the day considered (i.e., from hour 1 to 17 ) in the MILP model (Fig. 5(e)).

To gain better insight into the impacts of modeling linepack, we focus on hours 18-22 with comparatively low wind power production (Fig. 3). During these hours, the NGFPPs need to consume more natural gas to compensate the lack of wind power production. In the MISOCP model the increased need for natural gas during these hours is fully supplied by linepack, while keeping the gas supply profile still flat (Fig. 5(d)). Compared to the MISOCP model, linepack in the MILP model is not sufficient to fuel the increased power production of NGFPPs in hours 18 to 21 . Therefore, the natural gas supply is increased (Fig. 5(e)). Without modeling linepack, the natural gas consumption and supply need to be matched in each time period as illustrated in Fig. 5(f).

Based on the above results, the MISOCP model seems to reveal more linepack flexibility than the MILP model. However, compared to the MILP approximation, the MISOCP relaxation is less accurate in modeling the original constraints (1k). For both convexification models, we check the potential mismatch between the values obtained for the right- and left-hand sides of each equality constraint $(1 \mathrm{k})$. We quantify this mismatch using the normalized root mean squared error (NRMSE) with respect to the average flow. This error is $0.95 \%$ in the MISOCP model, while it is $0.03 \%$ only in the MILP model. A potential interpretation for the lower accuracy of the MISOCP model is that we used one relaxation technique (i.e., McCormick) within the process to achieve another relaxation, i.e., SOC constraints.

Regarding the computational aspects of the two models, the MISOCP model is solved on average within 10 seconds, while the MILP model requires 1,000 seconds to be solved with an optimality gap tolerance of $0.02 \%$. This implies that there is a trade-off between computational efficiency and accuracy of the flow representation in the two convexification approaches.

\section{CONCLusion}

This paper proposed a new MISOCP model for efficiently solving a combined power and natural gas dispatch problem, while accounting for both linepack and bidirectional flow in pipelines. The performance of the proposed model was compared against three models: (i) a similar problem but with MILP approximations of the linepack, (ii) a model neglecting the linepack, and (iii) a benchmark model with an ideal electric storage. Our results showed that the proposed model can be solved faster than the MILP model, but is less accurate. As potential future works, it is of interest to improve the accuracy of the MISOCP model by tightening 


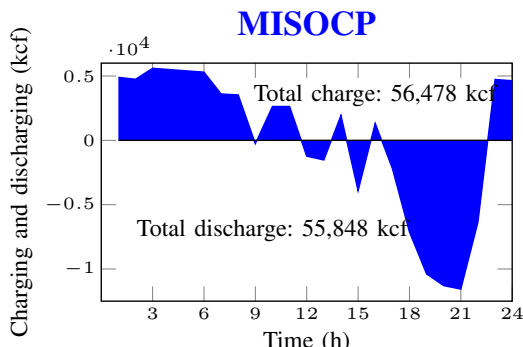

(a)

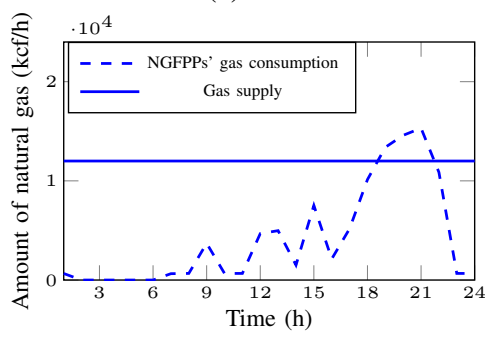

(d)

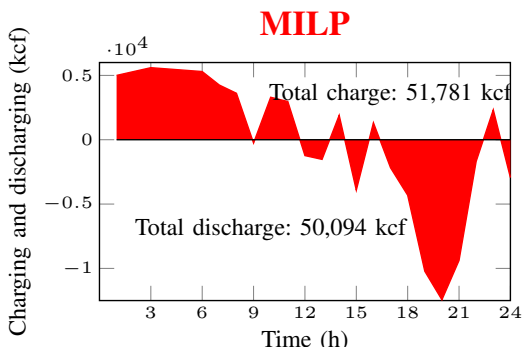

(b)

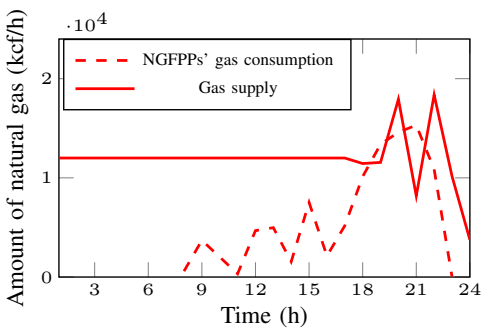

(e)

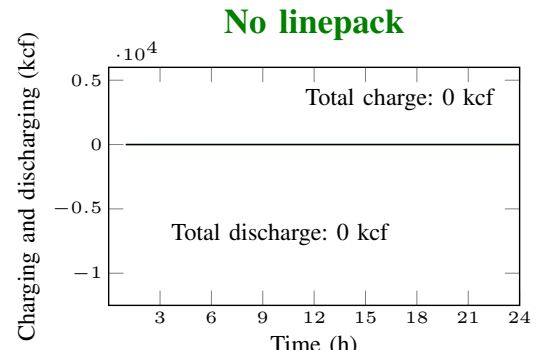

(c)

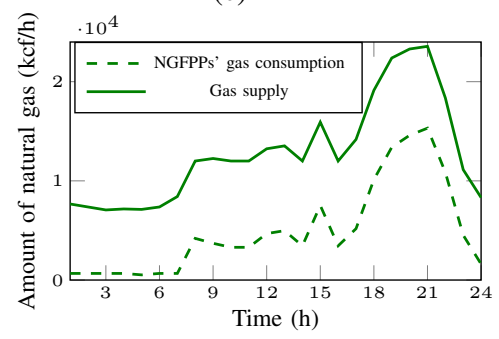

(f)

Fig. 5. Comparison of the results obtained from convexification approaches over 24 hours for $50 \%$ wind penetration. Plots (a) and (d) correspond to the MISOCP model, illustrating the hourly charging/discharging profile of linepack storage and the hourly profile of natural gas supply/consumption, respectively. The similar results for the MILP model are presented in plots (b) and (e), while they are depicted in plots (c) and (f) for a case ignoring linepack

McCormick relaxation [23], to investigate the possibility of adding sufficient conditions to tighten the SOC relaxation [18], and to explore alternatives for ex-post feasibility recovery [24].

\section{ACKNOWLEDGMENT}

This work was supported by the Danish Energy Technology Development and Demonstration Programme (EUDP) through the CORE project (12500/EUDP).

\section{REFERENCES}

[1] J. Gil, A. Caballero, and A. J. Conejo, "Power cycling: CCGTs: The critical link between the electricity and natural gas markets," IEEE Power and Energy Magazine, vol. 12, no. 6, pp. 40-48, 2014.

[2] M. Gil, P. Dueñas, and J. Reneses, "Electricity and natural gas interdependency: Comparison of two methodologies for coupling large market models within the European regulatory framework," IEEE Transactions on Power Systems, vol. 31, no. 1, pp. 361-369, 2016.

[3] C. M. Correa-Posada and P. Sanchez-Martin, "Security-constrained optimal power and natural-gas flow," IEEE Transactions on Power Systems, vol. 29 , no. 4, pp. 1780-1787, 2014.

[4] A. Antenucci and G. Sansavini, "Gas-constrained secure reserve allocation with large renewable penetration," IEEE Transactions on Sustainable Energy, vol. 9, no. 2, pp. 685-694, 2018.

[5] S. Clegg and P. Mancarella, "Integrated electrical and gas network flexibility assessment in low-carbon multi-energy systems," IEEE Transactions on Sustainable Energy, vol. 7, no. 2, pp. 718-731, 2016.

[6] C. Ordoudis, P. Pinson, and J. M. Morales, "An integrated market for electricity and natural gas systems with stochastic power producers," European Journal of Operational Research, vol. 272, no. 2, pp. 642654, 2019.

[7] C. M. Correa-Posada and P. Sánchez-Martín, "Integrated power and natural gas model for energy adequacy in short-term operation," IEEE Transactions on Power Systems, vol. 30, no. 6, pp. 3347-3355, 2015.

[8] A. Zlotnik, L. Roald, S. Backhaus, M. Chertkov, and G. Andersson, "Coordinated scheduling for interdependent electric power and natural gas infrastructures," IEEE Transactions on Power Systems, vol. 32, no. 1, pp. 600-610, 2017.

[9] C. Ordoudis, S. Delikaraoglou, P. Pinson, and J. Kazempour, "Exploiting flexibility in coupled electricity and natural gas markets: A price-based approach," in IEEE PowerTech, Manchester, UK, 2017.

[10] R. D. Tabors, S. Englander, and R. Stoddard, "Who's on first? The coordination of gas and power scheduling," The Electricity Journal, vol. 25 , no. 5 , pp. $8-15,2012$.
[11] P. Pinson, L. Mitridati, C. Ordoudis, and J. Ostergaard, "Towards fully renewable energy systems: Experience and trends in denmark," CSEE Journal of Power and Energy Systems, vol. 3, no. 1, pp. 26-35, 2017.

[12] A. Zlotnik, A. Rudkevich, R. Carter, P. Ruiz, S. Backhaus, and J. Tafl, "Grid architecture at the gas-electric interface," Los Alamos Natl. Lab., Santa Fe, NM, USA, Rep. LA-UR-17-23662, 2017.

[13] L. Bai, F. Li, T. Jiang, and H. Jia, "Robust scheduling for wind integrated energy systems considering gas pipeline and power transmission $\mathrm{N}-1$ contingencies," IEEE Transactions on Power Systems, vol. 32, no. 2, pp. 1582-1584, 2017

[14] S. D. Manshadi and M. E. Khodayar, "Coordinated operation of electricity and natural gas systems: A convex relaxation approach," IEEE Transactions on Smart Grid, to be published, 2018.

[15] Y. He, M. Shahidehpour, Z. Li, C. Guo, and B. Zhu, "Robust constrained operation of integrated electricity-natural gas system considering distributed natural gas storage," IEEE Transactions on Sustainable Energy, vol. 9, pp. 1061-1071, 2018.

[16] C. Borraz-Sánchez, R. Bent, S. Backhaus, H. Hijazi, and P. V. Hentenryck, "Convex relaxations for gas expansion planning," INFORMS Journal on Computing, vol. 28, no. 4, pp. 645-656, 2016.

[17] C. B. Sanchez, R. Bent, S. Backhaus, S. Blumsack, H. Hijazi, and P. Van Hentenryck, "Convex optimization for joint expansion planning of natural gas and power systems," in 2016 49th Hawaii International Conference on System Sciences (HICSS). IEEE, 2016, pp. 2536-2545.

[18] M. K. Singh and V. Kekatos, "Natural gas flow equations: Uniqueness and an MI-SOCP solver," arXiv preprint arXiv:1809.09025, 2018.

[19] C. Shao, X. Wang, M. Shahidehpour, X. Wang, and B. Wang, "An MILP-based optimal power flow in multicarrier energy systems," IEEE Transactions on Sustainable Energy, vol. 8, no. 1, pp. 239-248, 2017.

[20] L. Mitridati and J. A. Taylor, "Power systems flexibility from district heating networks," in 2018 Power Systems Computation Conference (PSCC), Dublin, Ireland, 2018.

[21] G. P. McCormick, "Computability of global solutions to factorable nonconvex programs: Part I - Convex underestimating problems," Mathematical Programming, vol. 10, no. 1, pp. 147-175, 1976.

[22] A. Schwele, C. Ordoudis, J. Kazempour, and P. Pinson, "Electronic companion: Coordination of power and natural gas systems: Convexification approaches for linepack modeling," 2018. [Online]. Available: https://zenodo.org/badge/latestdoi/161356114

[23] M. Bynum, A. Castillo, J.-P. Watson, and C. D. Laird, "Tightening McCormick relaxations toward global solution of the ACOPF problem," IEEE Transactions on Power Systems, to be published, 2018.

[24] L. Halilbasic, P. Pinson, and S. Chatzivasileiadis, "Convex relaxations and approximations of chance-constrained AC-OPF problems," IEEE Transactions on Power Systems, to be published, 2018. 